Article

\title{
Universal Power Flow Algorithm for Bipolar Multi-Terminal VSC-HVDC
}

\author{
Zhou Li ${ }^{1, *(D)}$, Yan He ${ }^{2}$, Ting-Quan Zhang ${ }^{1}$ and Xiao-Ping Zhang ${ }^{3}$ \\ 1 School of Electrical Engineering, Southeast University, Nanjing 210096, China; 220192788@seu.edu.cn \\ 2 Nanjing Power Supply Company of State Grid Jiangsu Electric Power Co., Ltd., Nanjing 210019, China; \\ 11100032147@jsepc.com.cn \\ 3 Department of Electronic Electrical and Systems Engineering, School of Engineering, University of \\ Birmingham, Birmingham B15 2TT, UK; x.p.zhang@bham.ac.uk \\ * Correspondence: lizhou@seu.edu.cn
}

Received: 18 December 2019; Accepted: 21 February 2020; Published: 26 February 2020

\begin{abstract}
An effective and accurate power flow algorithm provides control references for active power dispatch and initial steady state operating points, used for stability analysis, short-circuit calculations, and electromagnetic transient simulations, which is not only a fundamental precondition to analyze the system operating conditions, but also the basis to improve the accuracy of power flow and DC voltage control of the multi-terminal voltage source converter-based high voltage direct current (VSC-HVDC). This paper proposes a nodal voltage-based universal steady-state power flow algorithm for the newly-developed bipolar multi-terminal VSC-HVDC (VSC-MTDC). Firstly, as the positive-pole and negative-pole DC network of the bipolar VSC-MTDC can be operated individually, a bipolar power flow alternating iterative method is proposed here to obtain the positive/negative-pole DC network power flow. Secondly, a series of nodal equivalent methods involving various control strategies are proposed for the universal power flow algorithm. Then the detailed calculation procedure and a general MATLAB(TM) program for the universal power flow algorithm is presented. A typical 4-terminal bipolar VSC-MTDC system was built in the PSCAD/EMTDC to verify the validity of the proposed algorithm, and the results are discussed here. Moreover, the calculation results of more complex bipolar VSC-MTDC systems under different operating conditions, employing the proposed universal power flow algorithm, are presented to illustrate its universality and efficiency.
\end{abstract}

Keywords: universal power flow algorithm; multi-terminal; VSC-HVDC; bipolar configuration; alternating iterative method

\section{Introduction}

The advances in power electronic technology and the need for more flexible power transmission systems have led to the development of voltage source converter-based high voltage direct current (VSC-HVDC) transmission technology. It is a favorite solution for asynchronous grid interconnection, wind power integration, offshore platform power supply and urban power supply [1]. For the demand of various applications, the newly-developed bipolar VSC-HVDC system has the following novel features and characteristics:

(1) Availability of different DC network topologies. One obvious advantage of VSC-HVDC technology is that it can be extended to multi-terminal VSC-HVDC systems (VSC-MTDC) with radial, circular or meshed topology. In addition, it can facilitate the combination of decentralized generation units with large energy consumption centers. The merit of VSC-MTDC is driving real projects all over the world, such as the Tres Amigas Superstation project [2,3] and the Zhoushan 5-terminal MTDC project [4,5]. 
(2) Bipolar configuration. Due to the growing demand for transmission capacity, flexibility and reliability, the bipolar wiring mode supporting higher voltage level and larger transmission capacity in VSC-MTDC has gradually been applied in real projects [6]. As the positive-pole and negative-pole DC network of a VSC-MTDC system with bipolar wiring mode can be operated individually, it presents enhanced performance compared to traditional monopolar VSC-MTDC in operating flexibility and reliability $[7,8]$.

(3) Varieties of control strategies. With the integration of renewable energy via VSC-MTDC and the diversity in DC network topology, many improvements have been proposed in the system-level coordinated control strategies. Besides the master-slave control and DC voltage droop control [9-12] other improved coordinated control strategies involving amplitude-phase control [13-16] and multi-point voltage control [17] have been proposed to cope with different operating conditions.

These characteristics mentioned above increase the complexity in the operation of the VSC-MTDC system. On the other hand, they enable more flexible power transmission and dispatch with appropriate control strategies. An effective and accurate power flow algorithm, which can consider the newly developed bipolar VSC HVDC system and different varieties of control strategy, is needed to provide control reference for the active power dispatch, and hence get insights into the flexibility of bipolar VSC HVDC.

As power flow algorithms for VSC-MTDC systems have been investigated previously:

(1) A lot of publications have been focused on power flow studies on VSC-HVDC systems with symmetrical configurations $[18,19]$. However, there has been no prior work on power flow models and algorithms for the newly-developed VSC-MTDC with bipolar configuration considering the flexibility of the bipolar configuration, so that the individual power flows of the positive-pole and negative-pole DC networks can be implemented separately.

(2) Some publications have focused on control strategies. Some other publications were mainly focused on the power flow analysis of the VSC-MTDC system, and a series of specified algorithms based on Newton's classic method can be proposed to calculate the power flow for the VSC-MTDC under master-slave control strategy or DC voltage droop control strategy [20-22]. However, some other control strategies for VSC-MTDC have not been fully discussed, and unified calculation algorithms suitable for various control strategies has not been proposed.

(3) Other publications have focused on the flexibility of VSC-MTDC systems and the effects of different control strategies on the flexibility. Previous publications have mainly focused on the unified analysis algorithm for the interactions between AC grids and DC grids [19,23-25]. For the DC grid of VSC-MTDC, the converter power limits and losses were considered. In [23], different control modes of VSC HVDC were discussed. The DC grids including VSC converters are mainly seen as the extension of the AC grids. However, there are very few publications focusing on the flexibility of VSC-MTDC and the impacts of different control strategies on the flexibility. Thus, the impacts on the DC power flows of the various control strategies of VSCs, as well as the structures and topologies of the DC grid, have not been fully discussed.

Considering the reasons mentioned above, the aim of this paper was to develop a universal power flow algorithm for the newly-developed bipolar VSC-MTDC where the bipolar configuration is fully explored, and different structures and topologies, as well as both new and existing control strategies, are implemented.

Moreover, it is worth mentioning that the accuracy and efficiency of the DC power flow algorithm proposed in previous publications are verified only by comparison with the existing classic methods. A more convincing benchmark simulation system has been established in PSCAD, to verify the accuracy and efficiency of the proposed DC power flow algorithm.

This paper is organized as follows: firstly, Section 2 analyses the new development and requirements of VSC-MTDC; Section 3 illustrates the universal power flow algorithm for the bipolar VSC-MTDC system, and presents the detailed calculation procedure; in Section 4, calculation results 
using the proposed universal power flow algorithm are analyzed to verify its accuracy and efficiency; and finally, the conclusions are given in Section 5.

\section{New Development and Requirements of VSC-MTDC}

\subsection{New Development of VSC-MTDC}

\subsubsection{Bipolar Configuration of VSC-MTDC}

The newly-developed bipolar VSC-MTDC system is shown in Figure 1a, while Figure $1 \mathrm{~b}$ shows the topology of one terminal (Terminal 1) of the bipolar VSC-MTDC. In the bipolar VSC-HVDC, the positive-pole subsystem and the negative-pole subsystem are structurally symmetrical, and fully decoupled on the DC side. Each converter station consists of two individual converters, with one connecting to the positive-pole network (VSC+) and the other connecting to the negative-pole network (VSC-). On the DC side, one ground point is designated to join the two subsystems, a metallic wire is set up as the neutral line to provide a return circuit for the positive-pole network and negative-pole network, and thus they can be operated independently via the neutral line. While on the AC side, via two parallel-connected transformers, the two individual converters are connected to the AC bus of point of common coupling (PCC) respectively.

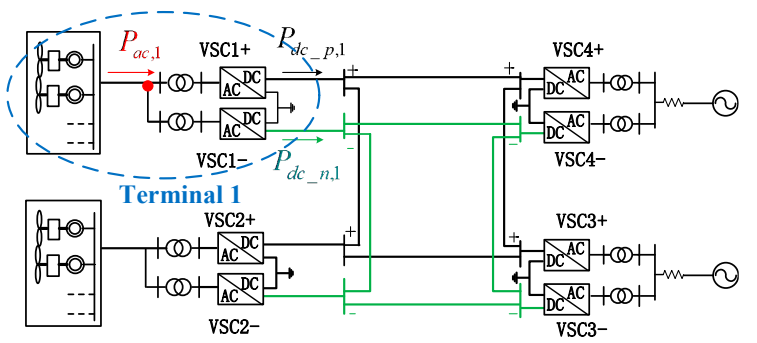

(a)

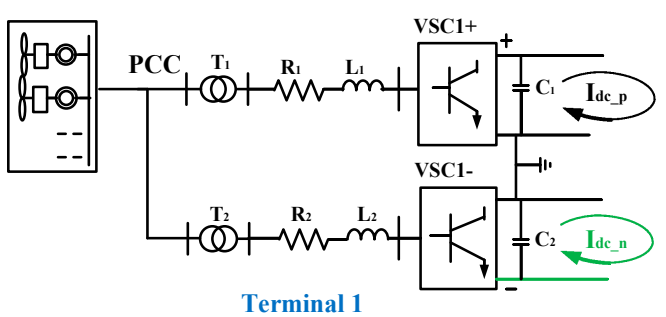

(b)

Figure 1. A bipolar multi-terminal voltage source converter-based high voltage direct current (VSC-MTDC) system. (a) The topology of a bipolar VSC-MTDC; (b) Structure of one terminal of a bipolar VSC-MTDC.

Hence, due to the newly-developed bipolar configuration, the positive-pole and negative-pole DC networks of the 4-terminal bipolar VSC-MTDC, as shown in Figure 1a, can be operated symmetrically or individually, depending on the VSC-MTDC system's situation. This presents an enhanced performance over traditional monopolar VSC-MTDC in operating flexibility and reliability. However, more complicated control strategies for bipolar VSC-MTDC are needed.

\subsubsection{Development of Control Strategies for VSC-MTDC}

With the development of VSC-MTDC, its applications have become diverse and various. Besides in AC grid interconnection, this technology is widely used in large-scale renewable energy integration, such as wind power integration, offshore platform power supply, and urban power supply. Furthermore, the topology of VSC-MTDC, including radial, circular, and meshed topologies, will become more and more complex in the future.

As a result, besides traditional control strategies, such as master-slave control and DC voltage droop control strategies [9-12], various station-level and system-level control strategies, such as amplitude-phase control [13-16] and multi-point voltage control [17] strategies, have been newly proposed to cope with different operating conditions. 


\subsection{Requirments of the Newly Developed VSC-MTDC}

Firstly, in order to realize the advantages of flexibility and reliability of the bipolar VSC-HVDC system, the positive-pole and negative-pole DC networks need to be operated individually. And the key basis of bipolar individual operations is to obtain the individual power flow of positive-pole and negative-pole DC network. However, there has been no prior work on power flow models and algorithms for the newly-developed VSC-MTDC with bipolar configuration, all of the existing DC power flow algorithms are only suitable for the monopolar or symmetrical operated bipolar VSC-MTDC system. Hence, a bipolar VSC-MTDC power flow algorithm method, which is valid to calculate the individual DC power flow of positive-pole and negative-pole operating under either symmetrical or asymmetric mode, is urgently needed.

Secondly, the development of different topologies and applications of VSC-MTDC systems has lead to the diversity of station-level and system-level control strategies for VSC-MTDC. In order to obtain the DC power flow, a universal power flow algorithm with wider feasibility of various control strategies under different operation conditions is in urgent need as well.

\section{Universal Power Flow Algorithm for the Newly Developed Bipolar VSC-MTDC}

In this section, considering the new requirements of VSC-MTDC, we propose the universal power flow algorithm, which has the following features: (1) different configurations, including monopolar/bipolar VSC-MTDC configuration, and different topologies including radial/circular/meshed DC networks; (2) different control strategies, including the coupled and decoupled control strategies; and (3) different bipolar VSC-MTDC operating conditions, such as symmetrical operating conditions, asymmetrical operating conditions.

\subsection{Main Structure of the Universal Power Flow Algorithm}

A universal power flow algorithm for VSC-MTDC with different topologies, different control strategies, and under different operating conditions, is proposed in this paper. Figure 2 shows the main structure of the proposed universal power flow algorithm:

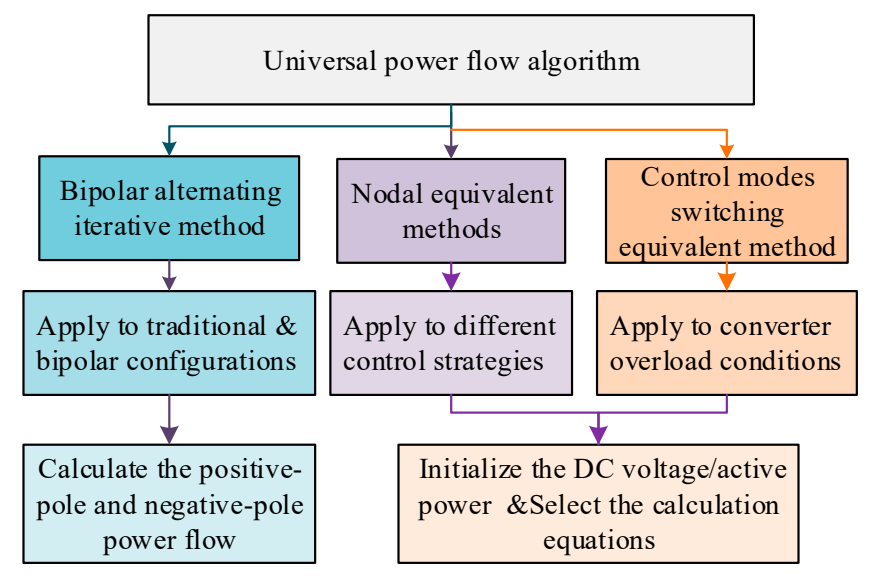

Figure 2. Block diagram of the universal power flow algorithm for VSC-MTDC.

Firstly, the bipolar alternating iterative method is applied to obtain the individual power flow of the positive-pole and negative-pole DC network, which makes the universal power flow algorithm suitable for the newly-developed VSC-MTDC with bipolar configuration.

Secondly, according to different control strategies used for the VSC-MTDC, including the coupled and decoupled control strategies, the nodal equivalent method is applied to decide the different initial DC voltage/active power and the composition of calculation equations for the universal power flow algorithm. 
And thirdly, in addition to obtain power flow for the VSC-MTDC under normal operating conditions, the control modes switching equivalent method as proposed in Section 3.4 is applied for the universal power flow algorithm to calculate the power flow under converter overload conditions, in which the control strategy of the overload converter should be changed and the voltage/active power references should be reassigned.

\subsection{Bipolar Power Flow Alternating Iterative Method}

\subsubsection{Nodal Voltage-Based Power Flow Algorithm}

The nodal voltage-based power flow algorithm is widely used to calculate the power flow of VSC-HVDC systems [20,23]. In a DC network of $n$ terminals, the overall current of node $i$ is equal to the sum of current injected into node $i$ from its adjacent nodes. The relationship between the voltage and the current can be expressed by Equation (1):

$$
\sum_{j=1}^{n} Y_{d c, i j} U_{d c, i}-\frac{P_{d c, i}}{U_{d c, i}}=0
$$

where $P_{d c, i}$ and $U_{d c, i}$ are the injected active power and DC voltage of node $i$, respectively; $Y_{d c, i j}$ is the element of the admittance matrix of the system.

Equation (1) is the basic equation for the nodal voltage-based power flow algorithm.

The form of admittance matrix $Y_{d c}$ follows Equation (2):

$$
\left\{\begin{array}{l}
Y_{d c, i j}=-y_{d c, i j} \\
Y_{d c, i i}=\sum_{j=1, j \neq i}^{n} y_{d c, i j}
\end{array}, i=1,2, \cdots n\right.
$$

where $y_{d c, i j}$ is the DC line admittance between node $i$ and node $j$.

Taking the 4-terminal VSC-MTDC in Figure $1 \mathrm{a}$ as an example, Figure 3 presents the nodal equivalent diagram of the system. Except the slack bus node 4, the active power injected into node 1, 2, 3 is a known variable before the calculation. Taking node 1 as an example, the overall active power injected into constant-P-control station 1 is given at every steady-state calculation, while the power distribution between positive-pole network $\left(P_{d c_{-}, p, 1}\right)$ and negative-pole network $\left(P_{d c_{-} n, 1}\right)$ relies on the topology and the situation of the VSC-MTDC system.

Under normal operating conditions, the bipolar topology is symmetrical, and the natural distribution of positive-pole and negative-pole DC networks should also be symmetrical. When fault appears on the monopole, positive-pole and negative-pole DC networks could be operated individually.

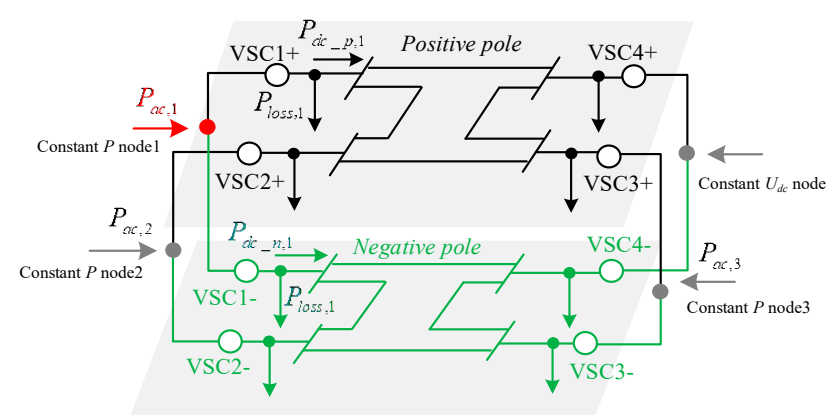

Figure 3. The nodal equivalent diagram of a bipolar VSC-MTDC. 


\subsubsection{Procedure of Bipolar Power Flow Alternating Iterative Methods}

For the newly-developed bipolar VSC-MTDC, the individual power flow for the positive-pole and negative-pole DC networks should be taken into consideration, and thus a bipolar power flow alternating iterative method is proposed in this paper.

Figure 4 shows the block diagram of the bipolar power flow alternating iterative method. The main principle of the method is to alternately iterate the positive-pole and negative-pole power injections. As the two individual converters are connected to the PCC on the AC side, the overall active power on the AC side is equal to the sum of positive-pole power and the negative-pole power. Thus in each iteration of the calculation the initial value of power injections of one pole should be updated by the power flow calculation results of the other pole and overall nodal active power, as is shown in Figure 4.

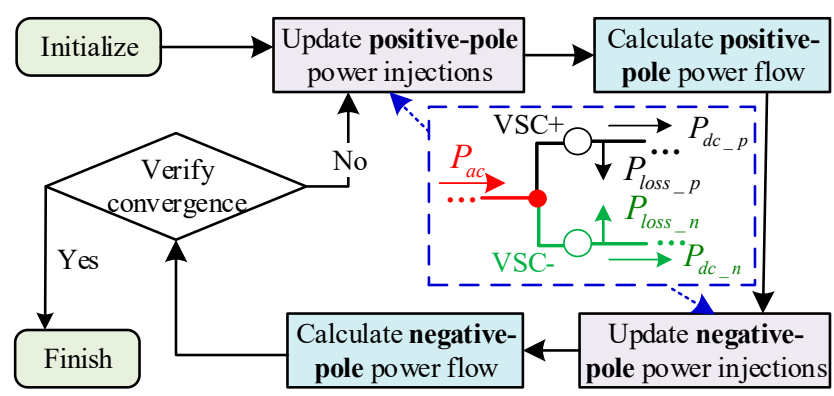

Figure 4. Bipolar power flow alternating iterative method.

Assume that $P_{a c 0}$ is the original overall nodal active power injections, whose element $P_{a c 0, i}$ is the nodal active power of Terminal $i$ :

$$
P_{a c 0}=\left[P_{a c 0,1}, P_{a c 0,2}, \ldots, P_{a c 0, n}\right]^{T}
$$

According to the paper of power loss consideration of VSC converter [26,27], the power loss of a VSC converter can be expressed as:

$$
P_{\text {loss }}=a+b I_{V S C}+c I_{V S C}^{2}
$$

$I_{V S C}$ and $P_{\text {loss }}$ are the current and power loss of the converter; $\mathrm{a}, \mathrm{b}$ and $\mathrm{c}$ are converter loss parameters which can be seen as constants presenting in [27], and the value of $a, b$ and $c$ will be given by Table 1 in Section 4 .

In each iteration of the calculations, the initial value of the positive-pole active power injections $P_{a c 0 \_p}^{(k)}$ and negative-pole active power injections $P_{a c 0 \_n}^{(k)}$ obey the following rules:

1. In the 1st iteration, $k=1$

$$
\left\{\begin{array}{l}
P_{d c 0 \_p}^{(k)}=\frac{1}{2} P_{a c 0}-P_{\text {loss_p }}^{(k)} \\
P_{d c 0 \_n}^{(k)}=\frac{1}{2} P_{a c 0}-P_{\text {loss } \_n}^{(k)}
\end{array}\right.
$$

The initial active power of each pole is assigned a half of the overall active power subtracts the converter loss.

2. In the kth iteration, $k \geq 2$

$$
\left\{\begin{array}{c}
P_{d c 0 \_p}^{(k)}=P_{a c 0}-\left(P_{d c \_n}^{(k-1)}+P_{\text {loss_n} n}^{(k-1)}\right)-P_{\text {loss_p }}^{(k)} \\
P_{d c 0 \_n}^{(k)}=P_{a c 0}-\left(P_{a c \_p}^{(k)}+P_{\text {loss_p }}^{(k)}\right)-P_{\text {loss_n }}^{(k)}
\end{array}\right.
$$


$P_{d c_{-} n}^{(k-1)}$ is the calculated negative-pole power in the $(k-1) t h$ iteration; $P_{d c_{-} p}^{(k)}$ is the calculated positive-pole power in the $k t h$ iteration.

$$
\left\{\begin{array}{l}
P_{\text {loss_p }}^{(k)}=a+b I_{p}^{(k)}+c I_{p}^{2(k)} \\
P_{\text {loss_n }}^{(k)}=a+b I_{n}^{(k)}+c I_{n}^{2(k)}
\end{array}\right.
$$

$P_{\text {loss_ } p}^{(k)}$ is the calculated positive-pole converter loss; $P_{\text {loss } \_n}^{(k)}$ is the calculated negative-pole converter loss; $I_{p}^{(k)}$ and $I_{n}^{(k)}$ are the currents of the converter of the positive-pole and negative pole respectively in the $k$ th iteration.

\subsection{Nodal Equivalent Methods}

\subsubsection{Various Control Strategies for VSC-MTDC and the Equivalent Relationships}

The key issue of the nodal voltage-based power flow algorithm is how to obtain the nodal active power and nodal voltage. Apparently, the difference in control strategies has influence on the specific composition of power flow equations.

The classification and characteristics of various control strategies for VSC-MTDC, from station level control strategies to system level control strategies, are summarized in Figure 5. As shown in Figure 5, the control of each VSC converter can be divided into coupled control, that is, amplified/phase control (A/F control) strategy and the active and reactive decoupled control ( $\mathrm{P} / \mathrm{Q}$ control) strategy. Moreover, for the $\mathrm{P} / \mathrm{Q}$ control strategy, it can be divided into voltage droop control and master-slave control strategy.

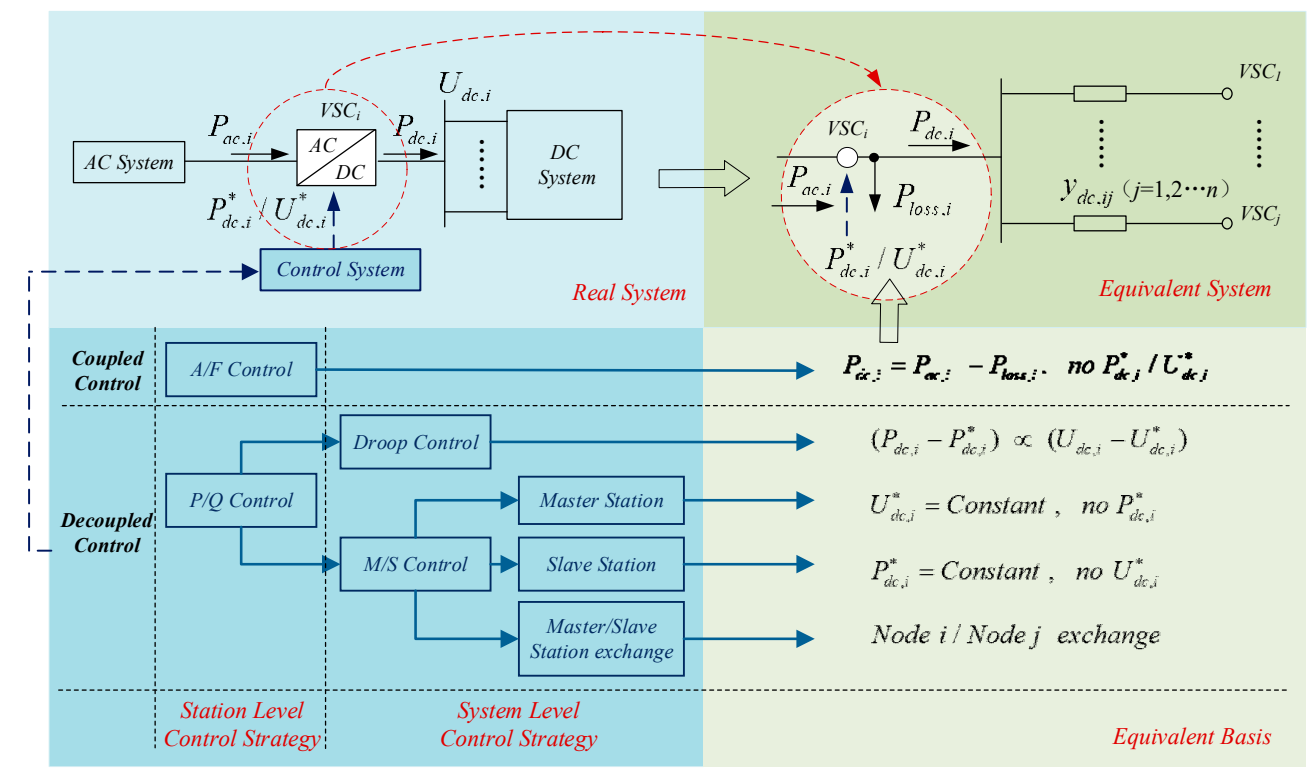

Figure 5. The equivalent relationships according to various control strategies for VSC-MTDC.

As shown in Figure 5, the known parameters (nodal power or nodal voltage) in the equivalent basis are different for different control strategies. A series of equivalence relationships of the DC voltages and active power with respect to particular control strategies can be seen in Figure 5. The formulation of power flow equations and the Jacobian matrix for different control strategies will be discussed in detail in Section 3.3.2. 


\subsubsection{Nodal Equivalent Method of Decoupled Control}

Previous power flow studies have been conducted on VSC-MTDC systems under the active power and reactive power decoupled control, which includes master-slave control and DC voltage droop control $[18,20,23]$.

As shown in Figure 5, for the master-slave control strategy, the master station adopts a constant DC voltage $\left(U_{d c}\right)$ control and the auxiliary stations adopt the constant active power $(P)$ control. For DC voltage droop control, as the voltage of the DC grid is controlled by several converters simultaneously, each node should be included in the power flow calculation equations. Then the nodal equivalent method of decoupled control can be summarized as follows:

- Constant $P$ control node

In the DC network of $n$ terminals, the active power of node 1 to node $n-1 P_{d c, 1}, P_{d c, 2}, \cdots, P_{d c, n-1}$ are known as constant parameters given by the power references of the constant active power control system, $U_{d c, 1}, U_{d c, 2}, \cdots, U_{d c, n-1}$ are unknown variables to be solved. Using Newton-Raphson power flow algorithm to solve Equation (1), where the Jacobian matrix can be expressed as:

$$
\left\{\begin{array}{l}
J_{d c, i i}=Y_{d c, i i}+P_{d c i} / U_{d c, j^{\prime}}^{2} i=1,2, \cdots, n-1 \\
J_{d c, i j}=Y_{d c, i j}, i \neq j
\end{array}\right.
$$

- Constant $U_{d c}$ control node

In the DC network of $n$ terminals, the DC voltage reference of this converter is constant, which is unique in the system to stabilize the DC voltage. Hence the DC voltage is a known parameter, the sequence number of the node is set $n$, and the node will not be involved in the equations set. This node can be seen as a slack bus to compensate the power imbalance of the overall DC grids. After the first $n-1$ nodal active power obtained according to Equation (1), the active power of node $n$ can be calculated as:

$$
P_{d c, n}=P_{d c, a l l}-P_{d c, 1}-P_{d c, 2}-\cdots-P_{d c, n-1}
$$

- $\quad$ DC voltage droop control node

According to the relationship between nodal active power and nodal voltage in DC voltage droop control strategy, the power flow calculation Equation (1) can be transformed to Equation (10):

$$
\sum_{j=1}^{n} Y_{d c, i j} U_{d c, i}-\frac{K_{d c, i}\left(U_{d c, i}^{*}-U_{d c i}\right)+P_{d c, i}^{*}}{U_{d c, i}}=0
$$

where $K_{d c, i}$ is the droop coefficient, $U_{d c, i}^{*}$ is the nodal DC voltage reference, $P_{d c, i}^{*}$ is the nodal active power reference.

Similarly, the Jacobian matrix can be expressed as:

$$
\left\{\begin{array}{l}
J_{d c, i i}=Y_{d c, i i}+\left(K_{d c, i} U_{d c, i}^{*}+P_{d c, i}^{*}\right) / U_{d c, j}^{2} \\
J_{d c, i j}=Y_{d c, i j}, i \neq j
\end{array}\right.
$$

\subsubsection{Nodal Equivalent Method of Coupled Control}

When the VSC-MTDC station is used for large scale renewable energy integration, the active power and reactive power decoupled control cannot be applied to the converter directly because the power conflict may occur between the power reference and the power output of renewable energy. Therefore, in this situation the coupled control, such as the amplitude-phase control, can be used to provide stable AC bus voltage for the renewable energy source. 
However, compared with the decoupled control, the equivalent method of coupled control has been paid little attention in existing publications. Hence, the nodal equivalent method of coupled control is proposed as follows. As shown in Figure 5, the equivalent method of the node operating under amplitude-phase control is similar to the constant $P$ node. In the steady-state power flow algorithm the active power of the VSCs, used for renewable energy integration, is treated as a known parameter whose value equals to the generating power of the renewable energy on the AC side. However, the parameter should be updated with the fluctuation of renewable energy generation. The $\mathrm{AC}$ active power, DC power and power loss of a VSC converter can be expressed as:

$$
\left\{\begin{array}{l}
P_{d c, i}=P_{a c, i}-P_{l o s s, i} \\
P_{l o s s, i}=a+b I_{V S C, i}+c I_{V S C, i}^{2}
\end{array}\right.
$$

where $P_{a c, i}$ is the given renewable energy generation power transmitted to the AC bus at Terminal $i, P_{d c, i}$ is the active power of node $i, I_{V S C, i}$ and $P_{l o s s, i}$ are the current and power loss of converter at Terminal $i$.

\subsection{Control Mode Switching Equivalent Method}

For the feasibility of the universal power flow algorithm under different operating conditions, abnormal conditions such as converter overload should be taken into consideration. For the system-level control strategy of VSC-MTDC, if any converter is overloaded, the control mode of the corresponding converter should be switched to an alternative control mode immediately. Thus, it may affect the power flow of the VSC-MTDC. Assume that the power flow converges after $k$ iterative calculations, the power and current limits should not be violated, which are given as follows:

$$
\left\{\begin{array}{l}
P_{d c \_ \text {min }, i} \leq P_{d c, i} \leq P_{d c_{-} \text {max }, i} \\
\frac{P_{d c, i}}{U_{d c, i}}+\frac{P_{l o s s, i}}{U_{d c, i}} \leq I_{V S C \_ \text {max }, i}
\end{array}\right.
$$

where $U_{d c, i}$ is the voltage of node $i, P_{d c_{-} m i n, i}$ and $P_{d c_{-} \max , i}$ are the lower and upper limit of the converter transmitted power at Terminal $i, I_{V S C_{-} \text {max }, i}$ is the upper limit of the current through the converter at Terminal $i$.

If Equation (13) cannot be satisfied, the nodal power should be modified to the power limit and the control strategy of this converter is modified to the constant $P_{d c}$ control.

Particularly, in the master-slave control strategy, if the power or current of the constant $U_{d c}$ control converter (master station) exceeds their limits, the master station should be switched to standby master station. Thus the corresponding nodal equivalent methods of decoupled control should be changed as well. In other words, the equivalent node type of the master converter should switch from constant $U_{d c}$ control node to constant $P$ control node; while the equivalent node type of the standby master converter should switch from constant $P$ control node to constant $U_{d c}$ control node.

\subsection{The Calculation Procedure of the Universal Power Flow Algorithm}

The detailed calculation steps of the proposed algorithm are presented in Figure 6. The detailed steps are as follows:

Step 1: Set the original data of the networks. Initialize the admittance matrix of positive-pole network $Y_{d \__{\_} p}$ and negative-pole network $Y_{d c_{-} n}$ according to Equation (2); initialize the overall nodal active power matrix $P_{d c 0}$ according to Equation (3).

Step 2: Set iterative number $k=1$. and (7);

Step 3: Initialize the positive-pole power matrix $P_{d c_{-} p}^{(k)}$ according to Equation (5) or Equations (6) 
Initialize voltage $U_{d c_{\_} p}^{(k)}$ and $U_{d c_{-} n}^{(k)}$. Because the nodal current relation-based power flow calculation is not sensitive to the initial voltages, the magnitude of each nodal voltage is set to 1.0 p.u. in the first iteration.

Step 4: Calculate the Jacobian matrix $J_{d c \_p}^{(k)}$ of positive-pole network according to Equation (8) or Equation (11).

Step 5: Calculate the positive-pole power flow based on the nodal current relationship in Equation (14):

$$
\Delta I_{d c_{-}, i}=\sum_{j=1}^{n} Y_{d c_{-}, i j} U_{d c_{-}, i}-\frac{P_{d c_{-}, i}, i}{U_{d c_{-}, i}, i}, i=1,2, \ldots, n
$$

The power flow equation is solved by Newton-Raphson method to obtain the calculated nodal power matrix $P_{d \__{-} p^{\prime}}^{(k)}$ nodal voltage $U_{d c \_p}^{(k)}$ and nodal voltage corrections $\Delta U_{d c_{-} p}^{(k)}$. The detailed calculation equations are presented in Appendix A Equation (A1) to (A5).

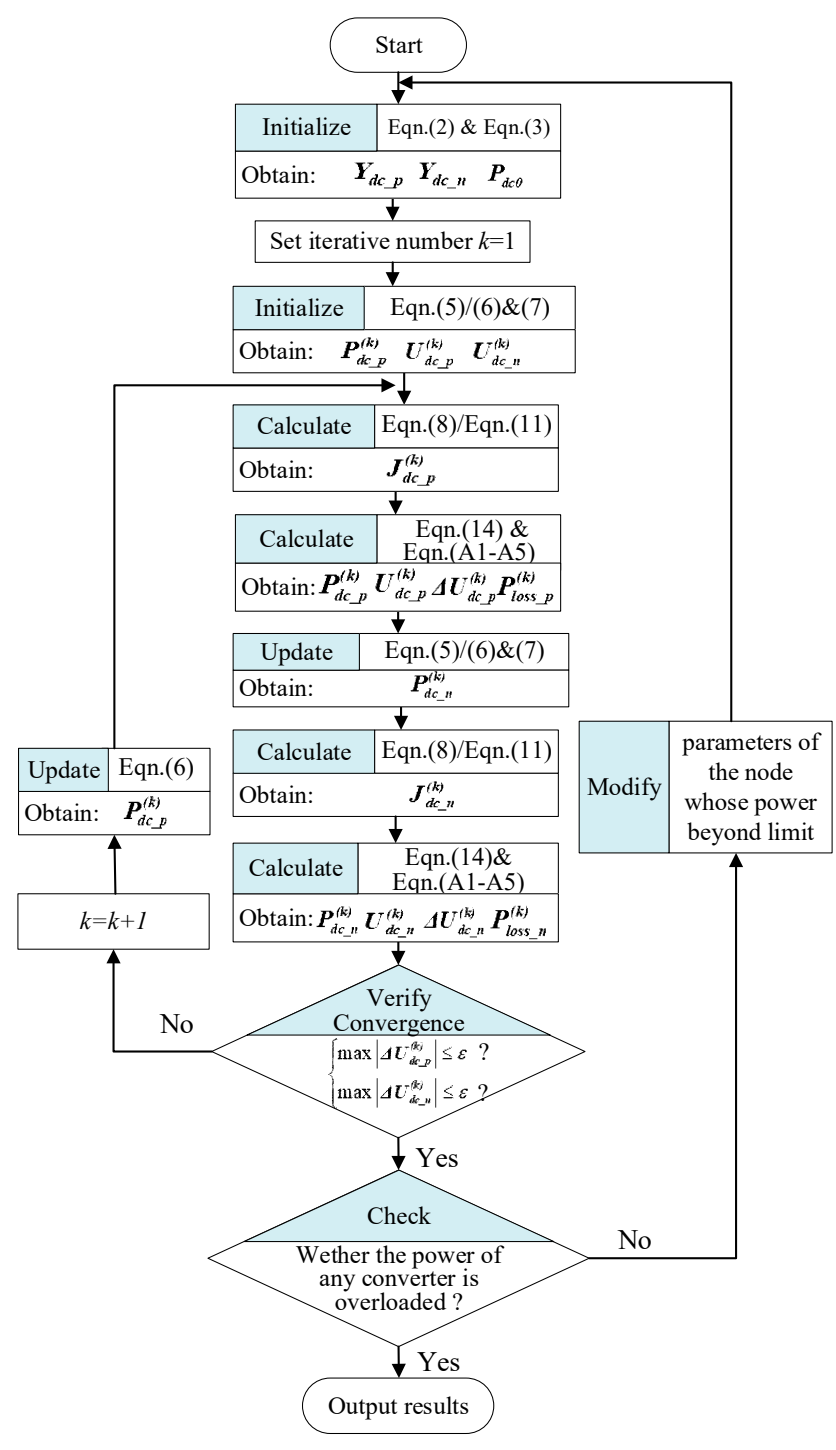

Figure 6. Power flow calculation procedure.

Step 6: Update the initial negative-pole power injections $P_{d c_{-} n}^{(k)}$ in the $k$ th iteration according to Equation (5) or Equations (6) and (7).

Step 7: Calculate the Jacobian matrix $J_{d c_{-} n}^{(k)}$ of negative-pole network according to Equation (8) or Equation (11). 
Step 8: Calculate the negative-pole power flow according to Equation (14), as well as Equation (A1) to (A5) in the Appendix A, to obtain the calculated nodal power injections $P_{d c_{-} n^{\prime}}^{(k)}$, nodal voltage $U_{d c_{-} n}^{(k)}$ and nodal voltage corrections $\Delta U_{d c_{-} n}^{(k)}$.

Step 9: Verify whether the maximum incremental change of the AC voltages and DC voltages are smaller than the convergent tolerance $\epsilon$.

$$
\left\{\begin{array}{l}
\max \left|\begin{array}{l}
\Delta U_{d c_{-} p}^{(k)} \\
\max \mid \Delta U_{d c_{-} n}^{(k)}
\end{array}\right| \leq \varepsilon
\end{array}\right.
$$

If Equation (15) is satisfied, skip to Step 10;

Otherwise, increase the iteration number $k=k+1$; At the same time, update the initial positive-pole power matrix according to Equation (5) and restart the positive-pole power flow calculation.

Step 10: Check whether power and current limits Equation (13) are not violated, output the power flow results and finish the iterations. Otherwise, go to Step 11.

Step 11: Modify the control strategy and parameters of the node whose transmitted power beyond limit. The initial active power of this node should be modified to the maximum or minimum value of the corresponding converter and form the overall nodal active power injections $P_{d c 0}$; form the admittance matrix $Y_{d c_{-} p}$ and $Y_{d c_{-} n}$ according to the modified parameters. Then go to Step 2.

\section{Case Study}

In order to verify the validity, universality and efficiency of the proposed universal steady-state power flow algorithm for the newly-developed VSC-MTDC were checked in MATLAB(TM). Then the universal steady-state power flow algorithm was tested by a typical 4-terminal, a more complex 6-terminal bipolar VSC-MTDC system and a larger 13-terminal bipolar system.

\subsection{Scenario1: 4-Terminal VSC-MTDC System}

The typical 4-terminal bipolar VSC-MTDC test system, as shown in Figure 7, is based on Chinese Zhangbei $\pm 500 \mathrm{kV}$ VSC-MTDC project [8]. The DC grid of which is with circular topology and bipolar wiring mode. Two (Terminal 1 and Terminal 2 ) of the four terminals are used for wind farms integration and operating under amplitude-phase control strategy, respectively; Terminal 3 is connected to an AC grid; and Terminal 4 is connected to a pumped storage power station, which serves as a balancing node of the system to stabilize the DC voltage. The base capacity of the system $\mathrm{S}_{\mathrm{B}}$ is $2000 \mathrm{MW}$ and the base voltage $\mathrm{U}_{\mathrm{B}}$ is $500 \mathrm{kV}$. The line resistance of the 4-terminal VSC-MTDC is shown in Table A1.

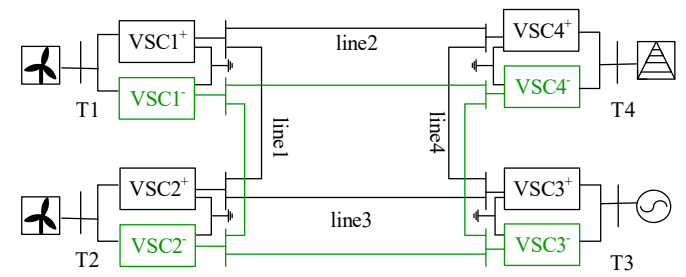

Figure 7. Topology diagram of 4-terminal VSC-MTDC.

As all the existing DC power flow algorithms are single-pole DC power flow algorithms, there is no pre-existing algorithm to be compared with the proposed bipolar VSC-MTDC power flow algorithm. Besides, previous papers usually compare their improved algorithm with a pre-existing algorithm to verify accuracy. However, the results of the pre-existing algorithm itself may not be accurate enough to be a benchmark.

Hence, in order to verify accuracy of the proposed algorithm in a more convincing way, the same 4-terminal bipolar VSC-MTDC system with detailed VSC electromagnetic transient models, 
as well as detailed control system, was also simulated in PSCAD/EMTDC as a benchmark system. The AC part of the simulation system was simplified to an ideal power source with the equivalent inductance. Meanwhile, in DC part, the detailed VSC converter model was built to verify various control strategies. Equivalent model parameters of the system and each converter station are listed in Table 1, corresponding control parameters for PI controllers are referred to in [7].

Table 1. The parameters of the AC-DC system model.

\begin{tabular}{ccc}
\hline Parameter & Symbol & Value \\
\hline Converter rated power & $P_{\text {rate }}(\mathrm{MW})$ & 3000 \\
VSC loss equivalent impedance & $Z_{e}($ p.u. $)$ & 1.0 \\
Rated frequency & $f_{e}(\mathrm{~Hz})$ & 50 \\
Rated AC voltage & $U_{a c}(\mathrm{kV})$ & 220 \\
Rated DC voltage & $U_{d c}(\mathrm{kV})$ & 500 \\
AC system equivalent inductance & $L_{a c}(\mathrm{mH})$ & 60 \\
Converter loss parameters & $\mathrm{a}$ & $11.033 \times 10^{-3}$ \\
& $\mathrm{~b}$ & $3.464 \times 10^{-3}$ \\
& $\mathrm{c}($ rectifier $)$ & $5.400 \times 10^{-3}$ \\
& $\mathrm{c}($ inverter $)$ & $7.670 \times 10^{-3}$ \\
\hline
\end{tabular}

The power flow results in the simulation platform were used as a benchmark to compare with the calculation results using the proposed universal steady-state power flow algorithm.

- Case 1: Single-point DC voltage control

In this case the VSC at Terminal 4 is operating at constant $U_{d c}$ control mode and the VSC at Terminal 3 is operating at constant $P$ control mode. The initial nodal voltage references are all assigned 1.0 p.u. in the general power flow calculation program. The initial nodal power flow references for each terminal (the summary of VSC+ and VSC-) are listed in Table A2.

At $6.0 \mathrm{~s}$, the active power of the load at Terminal 3 increases from 0.5 p.u. to 0.6 p.u. And at $10.0 \mathrm{~s}$, the active power of the wind farms at Terminal 1 raises from 0.25 p.u. to 0.35 p.u.

Since the positive-pole and negative-pole are symmetric, the calculated nodal active power and voltage results for bipolar networks in this algorithm are the same. Only the power flow for the positive-pole network is shown in Table 2.

Table 2. The monopolar calculated power flow results with the master-slave control (p.u.).

\begin{tabular}{cccccccccc}
\hline \multirow{2}{*}{$\mathbf{T}$} & \multicolumn{3}{c}{$\mathbf{5 - 6 ~ s}$} & \multicolumn{3}{c}{$\mathbf{6 - 1 0 ~ s}$} & \multicolumn{3}{c}{$\mathbf{1 0 - 1 5 ~ s}$} \\
\cline { 2 - 11 } & $\mathbf{U}_{\mathbf{d c}}$ & $\mathbf{P}_{\mathbf{d c}}$ & $\mathbf{P}_{\text {loss }}$ & $\mathbf{U}_{\mathbf{d c}}$ & $\mathbf{P}_{\mathbf{d c}}$ & $\mathbf{P}_{\text {loss }}$ & $\mathbf{U}_{\mathbf{d c}}$ & $\mathbf{P}_{\mathbf{d c}}$ & $\mathbf{P}_{\text {loss }}$ \\
\hline 1 & 1.0045 & 0.2378 & 0.0122 & 1.0041 & 0.2378 & 0.0122 & 1.0052 & 0.3372 & 0.0128 \\
2 & 1.0047 & 0.4364 & 0.0135 & 1.0041 & 0.4364 & 0.0136 & 1.0051 & 0.4365 & 0.0135 \\
3 & 0.9982 & -0.5113 & 0.0113 & 0.9972 & -0.6118 & 0.0118 & 0.9976 & -0.6118 & 0.0118 \\
4 & 1.0000 & -0.1590 & 0.0107 & 1.0000 & -0.0580 & 0.0109 & 1.0000 & -0.1564 & 0.0107 \\
\hline
\end{tabular}

The simulation curves in PSCAD/EMTDC and the results calculated by the calculation program are both plotted in Figure 8. By comparison, the calculated power flow is very close to the simulation results in the steady-state condition. 


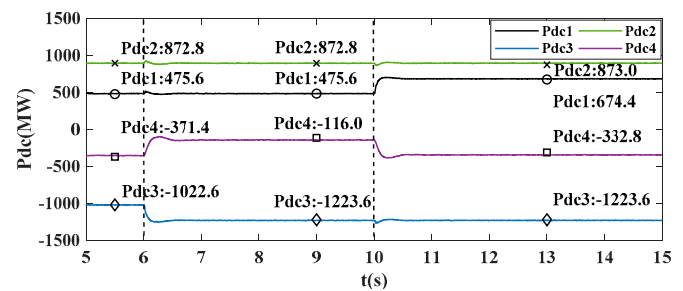

(a)

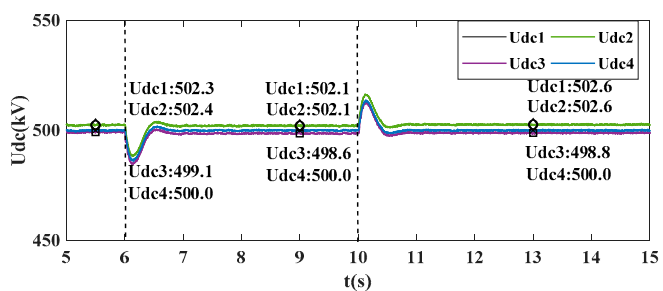

(b)

Figure 8. The simulation results in PSCAD. (a) Curves of the DC active power with the master-slave control; (b) Curves of the DC voltage with the master-slave control.

- Case 2: Multi-point DC voltage control

The converter station at Terminal 3 and Terminal 4 work at voltage droop control mode and their droop coefficient $K$ is set 40 . The initial active power references of each station are given in Table A3. The calculated results are given in Table 3 .

Table 3. The monopolar calculated power flow results with the multi-point voltage control (p.u.).

\begin{tabular}{cccccccc}
\hline $\mathbf{T}$ & \multicolumn{9}{c}{$\mathbf{5 - 6 ~ s}$} & $\mathbf{6 - 1 0 ~ s}$ & \multirow{K}{*}{$\mathbf{K}$} \\
\cline { 2 - 6 } & $\mathbf{U}_{\mathbf{d c}}$ & $\mathbf{P}_{\mathbf{d c}}$ & $\mathbf{P}_{\text {loss }}$ & $\mathbf{U}_{\mathbf{d c}}$ & $\mathbf{P}_{\mathbf{d c}}$ & $\mathbf{P}_{\text {loss }}$ & \\
\hline $\mathbf{1}$ & 1.0048 & 0.2294 & 0.0121 & 1.0076 & 0.3223 & 0.0127 & - \\
$\mathbf{2}$ & 1.0050 & 0.4325 & 0.0135 & 1.0077 & 0.4740 & 0.0138 & - \\
$\mathbf{3}$ & 0.9989 & -0.4545 & 0.0111 & 1.0005 & -0.5204 & 0.0113 & 40 \\
$\mathbf{4}$ & 1.0001 & -0.2036 & 0.0106 & 1.0018 & -0.2705 & 0.0107 & 40 \\
\hline
\end{tabular}

The simulation results in PSCAD/EMTDC and the calculated results are both plotted in Figure 9. With the wind power of converter station 1 increases from 0.2294 p.u.to 0.3223 p.u., and wind power of station 2 increase from 0.4325 p.u. to 0.4740 p.u. at $6.0 \mathrm{~s}$, the droop coefficient $K$ and power references of converter station 3 and 4 stay unchanged. As can be seen in Figure 9, with the increase of the wind power, the system DC voltages increase accordingly. In addition, the active power transmitted from Terminal 1,2 to Terminal 3,4, the voltage of the Terminal 1 and 2 is higher than that of Terminal 3 and 4 on the whole.

The calculated results are very close to the simulation results, which show the accuracy of the algorithm for the multi-point voltage control.

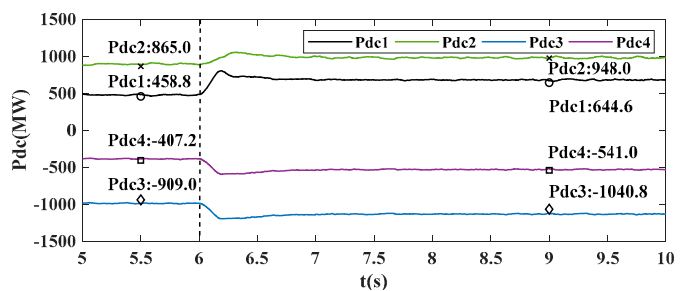

(a)

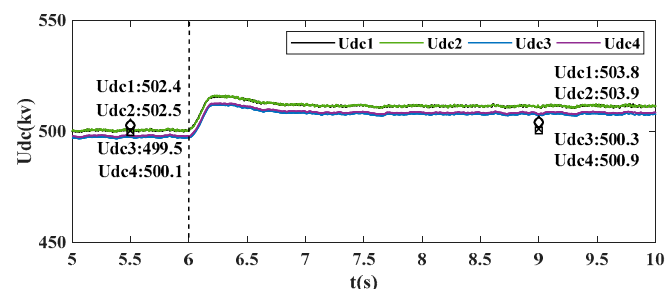

(b)

Figure 9. The simulation results in PSCAD. (a) Curves of the DC active power with the multi-point voltage control; (b) Curves of the DC voltage with the multi-point voltage control.

\subsection{Scenario2: 6-Terminal VSC-MTDC System}

In order to illustrate the universality and efficiency of the proposed universal steady-state power flow algorithm, it was further tested by a more complex 6-terminal bipolar VSC-MTDC system.

The test system is a meshed bipolar network connected to offshore wind farms by Terminal 1 and Terminal 2. Terminal 3, Terminal 4 and Terminal 5 are connected to AC grids. The Terminal 6 is 
connected to a pumped storage power station. The topology of the 6-terminal bipolar VSC-MTDC system is shown in Figure 10. The positive-pole network negative-pole network is symmetric and the monopolar line resistance is shown in Table A2. The base capacity of the system is $\mathrm{S}_{\mathrm{B}}=2000 \mathrm{MW}$ and the base voltage is $\mathrm{U}_{\mathrm{B}}=500 \mathrm{kV}$.

In the calculation program, the initial voltage reference and active power reference is given in Table A2. The iteration precision is $10^{-6}$.

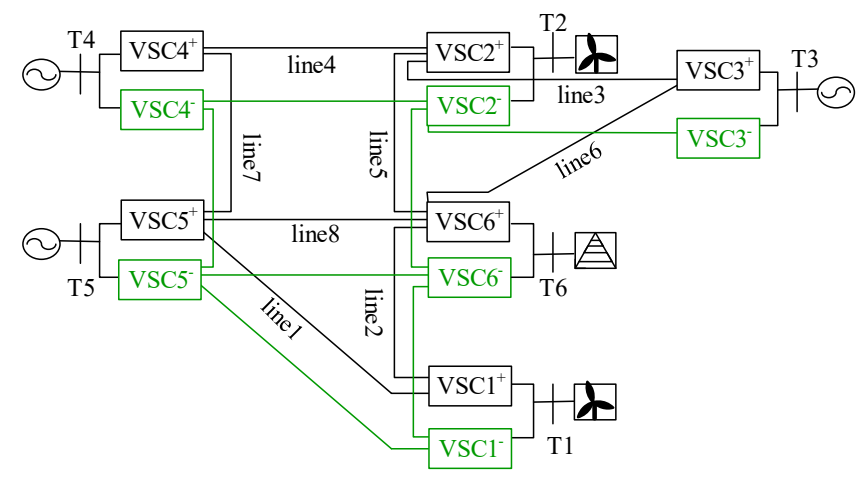

Figure 10. The topology of a 6-terminal bipolar VSC-MTDC.

\section{- $\quad$ Case 1: Single-point DC Voltage Control}

Converter station 6 is the balancing station, which uses constant $U_{d c}$ control to stabilize the system voltage. Among the other five converter stations, station 1, station 2 and station 3 use amplitude-phase control; station 4 and station 5 use constant $P$ control. The calculated DC voltage and active power of each VSC is shown in Table 4. $P_{d c 0, i}$ is the initial active power of each terminal. $P_{\max , i}$ is the maximum transmitted active power of each converter.

In this case, a disconnection fault happens to line 7 of the negative-pole network. Thus, the topology of the bipolar system is not symmetric.

The power flow of the positive-pole network and the negative-pole network can be calculated separately by the proposed alternating iterative method. Due to the topological asymmetry, there is a difference between the transmitted active power and DC voltage of two converters at the same terminal. The proposed algorithm can converge very quickly in only three iterations.

Table 4. Power flow calculation results in the single-point DC voltage control (p.u.).

\begin{tabular}{ccccccccc}
\hline \multirow{2}{*}{ Node } & \multirow{2}{*}{$\mathbf{P}_{\mathbf{a c} 0}$} & \multicolumn{3}{c}{ Positive Pole } & \multicolumn{3}{c}{ Negative Pole } & \multirow{2}{*}{$\mathbf{P}_{\max }$} \\
\cline { 3 - 8 } & & $\mathbf{U}_{\mathbf{d c}}$ & $\mathbf{P}_{\mathbf{d c}}$ & $\mathbf{P}_{\text {loss }}$ & $\mathbf{U}_{\mathbf{d c}}$ & $\mathbf{P}_{\mathbf{d c}}$ & $\mathbf{P}_{\text {loss }}$ & \\
\hline 1 & 0.45 & 1.0012 & 0.2129 & 0.0120 & 1.0005 & 0.2128 & 0.0120 & 0.5 \\
2 & 0.20 & 0.9973 & 0.0886 & 0.0114 & 0.9987 & 0.0886 & 0.0114 & 0.5 \\
3 & -0.25 & 0.9970 & -0.1357 & 0.0107 & 0.9977 & -0.1357 & 0.0107 & 0.5 \\
4 & -0.35 & 0.9944 & -0.1857 & 0.0107 & 0.9971 & -0.1857 & 0.0107 & 0.5 \\
5 & -0.30 & 0.9995 & -0.1607 & 0.0107 & 0.9984 & -0.1607 & 0.0107 & 0.5 \\
6 & 0.25 & 1.0000 & 0.1135 & 0.0115 & 1.0000 & 0.1135 & 0.0115 & 0.5 \\
\hline
\end{tabular}

\section{- $\quad$ Case 2: Multi-point DC voltage Control}

For multi-point DC voltage control, station 5 and station 6 work at voltage droop control mode to stabilize the system voltage cooperatively. The droop coefficient $K$ is set 40 . Besides, station 1 , station 2 and station 3 use amplitude-phase control. Station 4 uses constant $P$ control. The initial active power reference of each station is the same as those in Case 1. To further illustrate the individual operation of the bipolar network under different conditions, the positive-pole converter at Terminal 2 quits operation in this case. The calculated DC voltage and active power of each VSC is shown in Table 5. 
As shown in Table 5, the transmitted power through the positive-pole converter at Terminal 2 is 0 , and wind power supply ( 0.2 p.u.) for the grid goes through the negative-pole converter totally.

Therefore, the practical transmitted power on the positive-pole network is less than that on the negative-pole network. For station 5 and station 6 which use the DC voltage droop control, equal distribution between two converters is no longer exists; instead, the transmitted power distributed to the positive pole is less than that to the negative pole at Terminal 5 and 6 . At the same time, the positive-pole DC voltage at each terminal is lower than the negative-pole DC voltage.

Table 5. Power flow calculation results with multi-point voltage control (p.u.).

\begin{tabular}{ccccccccc}
\hline \multirow{2}{*}{ Node } & \multirow{2}{*}{$\mathbf{P}_{\text {ac0 }}$} & \multirow{K}{*}{} & \multicolumn{3}{c}{ Positive Pole } & \multicolumn{3}{c}{ Negative Pole } \\
\cline { 4 - 9 } & & & $\mathbf{U}_{\mathbf{d c}}$ & $\mathbf{P}_{\mathbf{d c}}$ & $\mathbf{P}_{\text {loss }}$ & $\mathbf{U}_{\mathbf{d c}}$ & $\mathbf{P}_{\mathbf{d c}}$ & $\mathbf{P}_{\text {loss }}$ \\
\hline 1 & 0.45 & - & 0.9997 & 0.2130 & 0.0120 & 1.0019 & 0.2130 & 0.0120 \\
2 & 0.20 & - & 0.9967 & 0.0000 & 0.0110 & 1.0009 & 0.1772 & 0.0118 \\
3 & -0.25 & - & 0.9961 & -0.1357 & 0.0107 & 0.9994 & -0.1357 & 0.0107 \\
4 & -0.35 & - & 0.9958 & -0.1857 & 0.0107 & 0.9989 & -0.1857 & 0.0107 \\
5 & -0.30 & 40 & 0.9979 & -0.0644 & 0.0108 & 1.0000 & -0.1482 & 0.0107 \\
6 & 0.25 & 40 & 0.9988 & 0.1740 & 0.0118 & 1.0011 & 0.0803 & 0.0113 \\
\hline
\end{tabular}

\subsection{Scenario3: 13-Terminal VSC-MTDC System}

In order to further validate the efficiency of the proposed universal steady-state power flow algorithm, a larger benchmark system [28] is selected and built into bipolar configuration for the algorithm testing.

The larger benchmark system is a 13-terminal meshed bipolar VSC-MTDC system, where Terminal 2, 5, 6, 9 and Terminal 11 are used for wind farms integration, while Terminal 1, 3, 4, 7, 8, 10, 12 and Terminal 13 are used for AC grids interconnection. The topology of the 13-terminal bipolar VSC-MTDC system is shown in Figure 11. The monopolar line resistance is given in Table A5, and the initial voltage reference and active power reference is given in Table A6.

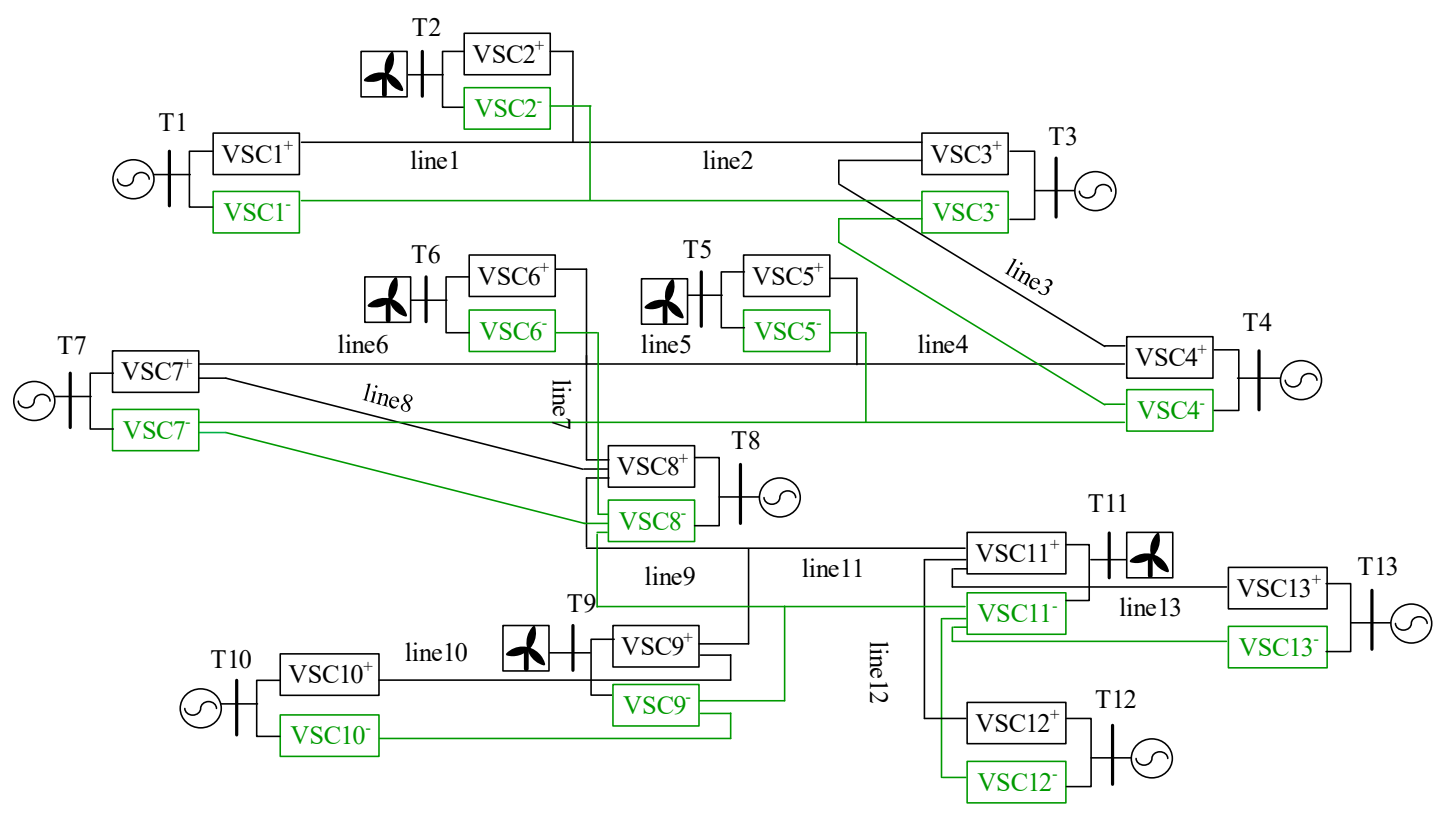

Figure 11. The topology of the larger scaled bipolar VSC-MTDC benchmark system.

In this case, converter station 13 is the balancing station, which uses constant $U_{d c}$ control to stabilize the system voltage. Among the other 12 converter stations, station 2, station 5 and station 6 use amplitude-phase control, the rest 9 stations use constant $P$ control. 
When the convergence tolerance is set to $10^{-6}$, the proposed universal power flow calculations can converge in only 5 iterations in both modes. The calculated DC voltage and active power of each VSC are shown in Table 6.

Table 6. Power flow calculation results with multi-point voltage control (p.u.).

\begin{tabular}{ccccccc}
\hline $\mathbf{T}$ & \multicolumn{3}{c}{ Mode1 (Iteration = 5) } & \multicolumn{3}{c}{ Mode2 (Iteration = 5) } \\
\cline { 2 - 6 } & $\mathbf{U}_{\mathbf{d c}}$ & $\mathbf{P}_{\mathbf{d c}}$ & $\mathbf{P}_{\text {loss }}$ & $\mathbf{U}_{\mathbf{d c}}$ & $\mathbf{P}_{\mathbf{d c}}$ & $\mathbf{P}_{\text {loss }}$ \\
\hline 1 & 0.9446 & -0.4917 & 0.0113 & 1.2112 & 0.2234 & 0.0119 \\
2 & 0.9738 & -0.0110 & 0.0110 & 1.2094 & 0.4867 & 0.0133 \\
3 & 0.9891 & 0.4909 & 0.0141 & 1.1852 & -0.2606 & 0.0106 \\
4 & 0.9895 & 0.4860 & 0.0140 & 1.1810 & -0.2450 & 0.0106 \\
5 & 0.9379 & -0.0110 & 0.0110 & 1.1630 & -0.4452 & 0.0131 \\
6 & 0.8877 & 0.0305 & 0.0112 & 1.1016 & 0.0098 & 0.0111 \\
7 & 0.8697 & -0.5117 & 0.0117 & 1.0918 & -0.1565 & 0.0107 \\
8 & 0.8840 & -0.4670 & 0.0114 & 1.0864 & -0.1371 & 0.0107 \\
9 & 0.9056 & -0.0110 & 0.0110 & 1.0831 & 0.4243 & 0.0132 \\
10 & 0.9009 & -0.0941 & 0.0108 & 1.0747 & -0.1982 & 0.0107 \\
11 & 0.9819 & 0.0720 & 0.0113 & 1.0285 & 0.0305 & 0.0111 \\
12 & 0.9904 & 0.2786 & 0.0124 & 1.0318 & 0.1135 & 0.0115 \\
13 & 1.0000 & 0.1699 & 0.0118 & 1.0000 & 0.1067 & 0.0115 \\
\hline
\end{tabular}

The results have validated the performance of the proposed algorithm, and thus have proven that the proposed algorithm can provide the active power dispatch solution and initial steady state operating points for stability analysis, short-circuit calculations and electromagnetic transient simulations as well as control references for the newly-developed bipolar VSC-MTDC systems.

\section{Conclusions}

This paper has proposed a nodal voltage-based universal steady-state power flow algorithm for the newly-developed bipolar VSC-MTDC system with different structures and topologies under various control strategies, which uses a bipolar power flow iterative method, and a series of nodal equivalent methods according to various control strategies.

The proposed universal power flow algorithm was designed in MATLAB(TM) and tested by a typical 4-terminal and a more complex 6-terminal as well as a 13-terminal bipolar VSC-MTDC. Simulation results in PSCAD/EMTDC have been presented as a reference to verify the validity of the proposed universal steady-state power flow algorithm:

- The proposed algorithm can be used to obtain the positive/negative-pole DC power flow, respectively, when the positive/negative-pole DC network of the newly-developed bipolar VSC-MTDC is operated individually.

- The proposed algorithm can be used to obtain the steady-state DC power flow of the VSC-MTDC with different topologies, including radial, circular, and meshed topologies. It is also applicable for the VSC-MTDC with various control strategies.

- The proposed algorithm can provide the active power dispatch solution and initial steady state operating points for stability analysis, short-circuit calculations and electromagnetic transient simulations, as well as control references for the newly-developed bipolar VSC-MTDC.

Author Contributions: Conceptualization, Z.L.; methodology, Z.L. and Y.H.; software, Y.H. and T.-Q.Z.; validation, Z.L., Y.H. and T.-Q.Z.; formal analysis, Z.L.; investigation, Z.L. and Y.H.; resources, Z.L.; data curation, Y.H. and T.-Q.Z.; writing — original draft preparation, Z.L. and Y.H.; writing-review and editing, Z.L., T.-Q.Z. and X.-P.Z.; visualization, Y.H. and T.-Q.Z.; supervision, X.-P.Z.; project administration, Z.L.; funding acquisition, Z.L. All authors have read and agreed to the published version of the manuscript.

Funding: This work was funded by the National Key Research and Development Program of China, grant number 2018YFB0904500 and the National Science Foundation of China, grant number 51707031. 
Conflicts of Interest: The authors declare no conflict of interest.

\section{Appendix A}

Table A1. The monopolar line resistance of the 4-terminal VSC-MTDC system.

\begin{tabular}{ccccc}
\hline Line Number & $\mathbf{1}$ & $\mathbf{2}$ & $\mathbf{3}$ & $\mathbf{4}$ \\
\hline Resistance $/ \Omega$ & 0.4940 & 2.0428 & 2.0557 & 1.8635 \\
\hline
\end{tabular}

Table A2. The initial nodal voltage and active power reference in the single-point voltage control (p.u.).

\begin{tabular}{ccccccc}
\hline \multirow{2}{*}{} & \multicolumn{2}{c}{$\mathbf{5 - 6} \mathbf{~ s}$} & \multicolumn{2}{c}{$\mathbf{6 - 1 0 ~} \mathbf{c}$} & \multicolumn{2}{c}{$\mathbf{1 0 - 1 5 ~ s}$} \\
\cline { 2 - 7 } & $\mathbf{U}_{\mathbf{d c}}$ & $\mathbf{P}_{\mathbf{d c} \mathbf{0}}$ & $\mathbf{U}_{\mathbf{d c}}$ & $\mathbf{P}_{\mathbf{d c} \mathbf{0}}$ & $\mathbf{U}_{\mathbf{d c}}$ & $\mathbf{P}_{\mathbf{d c} \mathbf{0}}$ \\
\hline $\mathbf{1}$ & 1.0000 & 0.4823 & 1.0047 & 0.4830 & 1.0042 & 0.3422 \\
$\mathbf{2}$ & 1.0000 & 0.8939 & 1.0049 & 0.8932 & 1.0042 & 0.4464 \\
$\mathbf{3}$ & 1.0000 & -1.0252 & 0.9983 & -1.2340 & 0.9972 & -0.6165 \\
$\mathbf{4}$ & 1.0000 & -0.3500 & 1.0000 & -0.1399 & 1.0000 & -0.1664 \\
\hline
\end{tabular}

Table A3. The initial nodal voltage and active power reference in the multi-point voltage control (p.u.).

\begin{tabular}{ccccc}
\hline \multirow{2}{*}{} & \multicolumn{2}{c}{$\mathbf{5 - 6} \mathbf{s}$} & \multicolumn{2}{c}{$\mathbf{6 - 1 0 ~ s}$} \\
\cline { 2 - 5 } & $\mathbf{U}_{\mathbf{d c}}$ & $\mathbf{P}_{\mathbf{d c 0}}$ & $\mathbf{U}_{\mathbf{d c}}$ & $\mathbf{P}_{\mathbf{d c} \mathbf{0}}$ \\
\hline $\mathbf{1}$ & 1.0000 & 0.4830 & 1.0000 & 0.6700 \\
$\mathbf{2}$ & 1.0000 & 0.8920 & 1.0000 & 0.9756 \\
$\mathbf{3}$ & 1.0000 & -1.0000 & 1.0000 & -1.0000 \\
$\mathbf{4}$ & 1.0000 & -0.4000 & 1.0000 & -0.4000 \\
\hline
\end{tabular}

Table A4. The monopolar line resistance of the 6-terminal VSC-MTDC system.

\begin{tabular}{ccccccccc}
\hline Line Number & $\mathbf{1}$ & $\mathbf{2}$ & $\mathbf{3}$ & $\mathbf{4}$ & $\mathbf{5}$ & $\mathbf{6}$ & $\mathbf{7}$ & $\mathbf{8}$ \\
\hline Resistance $/ \Omega$ & 1.5 & 2.0 & 3.0 & 2.0 & 3.0 & 3.0 & 2.0 & 3.0 \\
\hline
\end{tabular}

Table A5. The monopolar line resistance of the 13-terminal VSC-MTDC system.

\begin{tabular}{lcccccccc}
\hline Line Number & $\mathbf{1}$ & $\mathbf{2}$ & $\mathbf{3}$ & $\mathbf{4}$ & $\mathbf{5}$ & $\mathbf{6}$ & $\mathbf{7}$ & $\mathbf{8}$ \\
Resistance $/ \Omega$ & 6.0 & 3.0 & 0.6 & 6.0 & 6.0 & 2.4 & 2.4 & 4.0 \\
\hline Line Number & $\mathbf{9}$ & $\mathbf{1 0}$ & $\mathbf{1 1}$ & $\mathbf{1 2}$ & $\mathbf{1 3}$ & $\mathbf{1 4}$ & & \\
Resistance $/ \Omega$ & 2.8 & 2.4 & 2.4 & 9.6 & 1.6 & 2.4 & & \\
\hline
\end{tabular}

Table A6. The initial nodal voltage and active power reference of the 13-terminal VSC-MTDC system (p.u.).

\begin{tabular}{|c|c|c|c|c|c|c|c|c|}
\hline \multicolumn{2}{|c|}{$\mathbf{T}$} & 1 & 2 & 3 & 4 & 5 & 6 & 7 \\
\hline \multirow{2}{*}{ Mode1 } & $\mathrm{U}_{\mathrm{dc}}$ & 1.0000 & 1.0000 & 1.0000 & 1.0000 & 1.0000 & 1.0000 & 1.0000 \\
\hline & $\mathrm{P}_{\mathrm{dc}}$ & -0.9608 & 0.0000 & 1.0100 & 1.0000 & 0.0000 & 0.0833 & -1.0000 \\
\hline \multirow{2}{*}{ Mode2 } & $\mathrm{U}_{\mathrm{dc}}$ & 1.0000 & 1.0000 & 1.0000 & 1.0000 & 1.0000 & 1.0000 & 1.0000 \\
\hline & $\mathrm{P}_{\mathrm{dc}}$ & 0.4706 & 1.0000 & -0.5000 & -0.4678 & 0.9167 & 0.0417 & -0.2917 \\
\hline \multicolumn{2}{|c|}{$T$} & 8 & 9 & 10 & 11 & 12 & 13 & \\
\hline \multirow{2}{*}{ Mode1 } & $\mathrm{U}_{\mathrm{dc}}$ & 1.0000 & 1.0000 & 1.0000 & 1.0000 & 1.0000 & 1.0000 & \\
\hline & $\mathrm{P}_{\mathrm{dc}}$ & -0.9112 & 0.0000 & -0.1667 & 0.1667 & 0.5820 & 0.3633 & \\
\hline \multirow{2}{*}{ Mode2 } & $\mathrm{U}_{\mathrm{dc}}$ & 1.0000 & 1.0000 & 1.0000 & 1.0000 & 1.0000 & 1.0000 & \\
\hline & $P_{d c}$ & -0.2528 & 0.8750 & -0.3750 & 0.0833 & 0.2500 & 0.2363 & \\
\hline
\end{tabular}




$$
\begin{aligned}
& \Delta I_{d c_{\_} p}^{(k)}=\left[\Delta I_{d c_{\_} p, 1}, \Delta I_{d c_{\_}, 2,2}, \cdots, \Delta I_{d c_{\_}, p, n}\right]^{T} \\
& \Delta U_{d c_{-} p}^{(k)}=-J_{d c_{-} p}^{(k)-1} \cdot \Delta I_{d c_{-} p}^{(k)} \\
& U_{d c_{\_} p}^{(k)}=U_{d c_{\_} p}^{(k)}-\Delta U_{d c_{-} p}^{(k)} \\
& P_{d c_{-}, i, i}^{(k)}=U_{d c_{-}, i, i}^{(k)} \cdot I_{d c_{-} p, i^{\prime}}^{(k)} i=1,2, \ldots, n \\
& P_{d c_{\_} p}^{(k)}=\left[P_{d c_{-}, 1^{\prime}}^{(k)}, P_{d c_{-}, 2^{\prime}}^{(k)} \cdots, P_{d c_{-}, n, n}^{(k)}\right]^{T}
\end{aligned}
$$

\section{References}

1. Guo, C.; Zhao, C. Supply of an Entirely Passive AC Network Through a Double-Infeed HVDC System. IEEE Trans. Power Electron. 2010, 25, 2835-2841.

2. Reynolds, M.; Stidham, D.; Alaywan, Z. The Golden Spike: Advanced Power Electronics Enables Renewable Development Across NERC Regions. IEEE Power Energy Mag. 2012, 10, 71-78. [CrossRef]

3. Alaywan, Z. The Tres Amigas Superstation: Linking renewable energy and the nation's grid. In Proceedings of the IEEE PES General Meeting, Providence, RI, USA, 25-29 July 2010; pp. 1-5.

4. Gomis-Bellmunt, O.; Liang, J.; Ekanayake, J. Topologies of multiterminal HVDC-VSC transmission for large offshore wind farms. Electr. Power Syst. Res. 2011, 81, 271-281. [CrossRef]

5. Jie, Z.; Haibin, L.; Rui, X. Research of DC circuit breaker applied on Zhoushan multi-terminal VSC-HVDC project. In Proceedings of the 2016 IEEE PES Asia-Pacific Power and Energy Engineering Conference (APPEEC), Xi'an, China, 25-28 October 2016; pp. 1636-1640.

6. Guo, X.; Deng, M.; Wang, K. Characteristics and performance of Xiamen VSC-HVDC transmission demonstration project. In Proceedings of the 2016 IEEE International Conference on High Voltage Engineering and Application (ICHVE), Chengdu, China, 19-22 September 2016; pp. 1-4.

7. Li, Z.; He, Y.; Li, Y.; Gu, W.; Tang, Y.; Zhang, X.-P. Hybrid Control Strategy for AC Voltage Stabilization in Bipolar VSC-MTDC. IEEE Trans. Power Syst. 2019, 34, 129-139. [CrossRef]

8. Li, Z.; He, Y.; Li, Y. Active Power Shifting Strategy for the Bipolar VSC-MTDC. In IOP Conference Series: Earth and Environmental Science; IOP Publishing: Qingdao, China, 2018; Volume 223.

9. Beerten, J.; Cole, S.; Belmans, R. Modeling of multi-terminal VSC HVDC systems with distributed DC voltage control. IEEE Trans. Power Syst. 2013, 29, 34-42. [CrossRef]

10. Khan, S.; Bhowmick, S. A Comprehensive Power Flow Model of Multi-terminal PWM Based VSC-HVDC Systems with DC Voltage Drop Control. Int. J. Electr. Power Energy Syst. 2018, 102, 71-83. [CrossRef]

11. Raza, A.; Liu, Y.; Rouzbehi, K.; Jamil, M.; Gilani, S.O.; Xu, D.; Williams, B.W. Power Dispatch and Voltage Control in Multiterminal HVDC Systems: A Flexible Approach. IEEE Access 2017, 5, 24608-24616. [CrossRef]

12. Rouzbehi, K.; Miranian, A.; Luna, A.; Rodriguez, P. DC voltage control and power sharing in multiterminal DC grids based on optimal DC power flow and voltage-droop strategy. IEEE J. Emerg. Sel. Top. Power Electr. 2014, 2, 1171-1180. [CrossRef]

13. Li, H.; Liu, C.; Li, G.; Iravani, R. An Enhanced DC Voltage Droop-Control for the VSC-HVDC Grid. IEEE Trans. Power Syst. 2017, 32, 1520-1527. [CrossRef]

14. Abdel-Khalik, A.S.; Massoud, A.M.; Elserougi, A.A.; Ahmed, S. Optimum power transmission-based droop control design for multi-terminal HVDC of offshore wind farms. IEEE Trans. Power Syst. 2013, 28, 3401-3409. [CrossRef]

15. Hu, J.; Zhu, J.; Dorrel, D. Predictive direct power control of doubly fed induction generators under unbalanced grid voltage conditions for power quality improvement. IEEE Trans. Sustain. Energy 2014, 6, 687-695. [CrossRef]

16. Zhao, H.; Wu, Q.; Wang, J.; Liu, Z.; Shahidehpour, M.; Xue, Y. Combined active and reactive power control of wind farms based on model predictive control. IEEE Trans. Energy Convers. 2017, 32, 1177-1187. [CrossRef]

17. Li, Z.; Li, Y.; Zhan, R.; He, Y.; Zhang, X. AC Grids Characteristics Oriented Multi-Point Voltage Coordinated Control Strategy for VSC-MTDC. IEEE Access 2019, 7, 7728-7736. [CrossRef] 
18. Renedo, J.; García-Cerrada, A.; Rouco, L. Reactive-Power Coordination in VSC-HVDC Multi-Terminal Systems for Transient Stability Improvement. IEEE Trans. Power Syst. 2017, 32, 3758-3767. [CrossRef]

19. Bojorquez-Melendrez, E.; Ugalde-Caballero, L.E.; Moreno-Goytia, E.L. Sequential algorithm-based model for the study of AC/DC power flow in VSC-MTDC systems. In Proceedings of the 2015 IEEE International Autumn Meeting on Power, Electronics and Computing (ROPEC), Ixtapa, Mexico, 4-6 November 2015; pp. 1-5.

20. Wang, W.; Barnes, M. Power Flow Algorithms for Multi-Terminal VSC-HVDC with Droop Control. IEEE Trans. Power Syst. 2014, 29, 1721-1730. [CrossRef]

21. Simpson-Porco, J.W. Lossy DC Power Flow. IEEE Trans. Power Syst. 2018, 33, 2477-2485. [CrossRef]

22. Garcés, A. On the Convergence of Newton's Method in Power Flow Studies for DC Microgrids. IEEE Trans. Power Syst. 2018, 33, 5770-5777. [CrossRef]

23. Zhang, X.-P. Multiterminal Voltage-Sourced Converter Based HVDC Models for Power Flow Analysis. IEEE Trans. Power Syst. 2004, 18, 1877-1884. [CrossRef]

24. Yi, W.; Juanjuan, L.; Tong, Z.; Mingyu, Z.; Xin, S.; Jinjun, L. A unified power flow method of hybrid AC/DC power system with VSC-MTDC. In Proceedings of the 2017 IEEE Conference on Energy Internet and Energy System Integration (EI2), Beijing, China, 26-28 November 2017; pp. 1-5.

25. Beerten, J.; Cole, S.; Belmans, R. Generalized Steady-State VSC MTDC Model for Sequential AC/DC Power Flow Algorithms. IEEE Trans. Power Syst. 2012, 27, 821-829. [CrossRef]

26. Liang, H.; Zhao, X.; Yu, X.; Gao, Y.; Yang, J. Study of Power Flow Algorithm of AC/DC Distribution System including VSC-MTDC. Energies 2015, 8, 8391-8405. [CrossRef]

27. Renedo, J.; García-Cerrada, A.; Rouco, L.; Sigrist, L. Coordinated Control in VSC-HVDC Multi-Terminal Systems to Improve Transient Stability: The Impact of Communication Latency. Energies 2019, 12, 3638. [CrossRef]

28. An, T.; Zhou, X.; Han, C.; Wu, Y.; He, Z.; Pang, H.; Tang, G. A DC Grid Benchmark Model for Studies of Interconnection of Power Systems. CSEE J. Power Energy Syst. 2015, 1, 101-109. [CrossRef]

(C) 2020 by the authors. Licensee MDPI, Basel, Switzerland. This article is an open access article distributed under the terms and conditions of the Creative Commons Attribution (CC BY) license (http://creativecommons.org/licenses/by/4.0/). 